Semina $\square \quad \mathrm{Nr} 15$

Scientiarum 2016

s. $77-87$

DOI: http://dx.doi.org/10.15633/ss.1769

Adam Fedyniuk

\title{
Modular structurality \\ and emergent functionality \\ within knowledge representation systems
}

In contemporary science, at the crossroads between cognitive science and knowledge engineering one can find a research niche that can benefit substantially from interdisciplinary work. The discipline that concerns this particular area of scientific inquiry, and is mostly relevant to the subject of this paper is known as ontology metamodelling. Ontology metamodelling is a discipline that uses various tools to propose and test on different levels the viability of new and innovative approaches and architectures that may be applied to knowledge engineering. As is commonly stated, in ontology design there is no one right and true way of choosing the adequate tools and methodology. Some ideas are worthy of being tested in practical terms instantly when they are devised (due to level their level of detail and specificity), but some need a thorough analysis and strong conceptual framework as it's foundations to even be considered viable. That is one of the main goals of metamodelling in general: to check if those bold ideas, and inspirations can have a meaningful application as well as coherent theoretical structure. The necessity for it stems from the fact, that oftentimes some explanations and approaches in knowledge engineering may be too resource-dependent and in the end, time-consuming. In some ways this discipline may be considered akin to the practical considerations within the discourse of philosophy of information, and in many ways there can 
be seen many similarities in the areas of expertise. Yet, as it may seem, the philosophical approach emphasizes interdisciplinarity, and at times very strict scrutiny that may be applied to the theoretical framework of analysed solutions.

\section{Connection between interdisciplinary domains}

One of the major areas where one can find an inspiration for new ideas concerning the structurality found in artificial knowledge representation systems (such as domain ontologies in conjunction with their editors equipped with various reasoning engines) can be seen in biology. ${ }^{1}$ To further emphasize this connection, it is worthwhile to look at similarities between connectomics and proteomics. Both of these disciplines delve into the structurality of information processing, and both employ the same mathematical theories for the analysis of it's structures. Especially one seems to be viable in wide variety of subjects in the interdisciplinary work - centrality. A mathematical theory that is widely used in social network analysis, was proven useful both in the fields of proteomics and connectomics. ${ }^{2}$ Additionally, the structure of the content within various ontologies can be represented in network or graph format and that makes it also a subject to network analysis and it's structure prone to centrality measurement. ${ }^{3}$

With network structure in mind, it is far easier to consider creating a modular architecture for artificial knowledge representation systems. In many cases, such ideas are explored, but one must consider another side that contributes to innovative ontology de-

1 F. Azam, Biologically inspired modular neural networks, Blacksburg, VA 2000.

2 F. Cheng and others, Quantitative network mapping of the human kinome interactome reveals new clues for rational kinase inhibitor discovery and individualized cancer therapy, "Oncotarget" 15 (2014), p. 3697-3710, doi: 10.18632/oncotarget. 1984; Zuo X. N., R and others, Network centrality in the human functional connectome, "Cerebral Cortex" 22 (2012) pp. 1862-1875, doi: 10.1093/cercor/bhr269.

3 C. Roche, Network analysis of Semantic Web Ontologies, Stanford CS224W Social and Information Network Analysis 2011. 
sign - the biological structures that could be the meaningful inspiration for new approaches and solutions in knowledge engineering. In some way one can protest with saying that it contributes to reductionist claims and overall can be limiting for ontology designer, but it is possible that within biological systems we can find bizzare and hard to explain phenomena that may in future become paramount to meaningful application of ontologies. One of such phenomena, that is highly elusive and provoked a lot of discord between philosophers is the mechanism of emergence. In systems biology emergent functions can be found within various metabolic tracts. The flow of information between different protein domains creates a kinase network that exhibit emergent properties and functions, and as such they do so by maintaining a modular structure, that changes it's pathways in response to damage and maintains the stability of information flow, sometimes by being supported by parts of the network that normally takes part in enabling completely different function. The modular structure of those networks clearly is an appropriate foundation for those emergent properties. One can infer that if we are able to duplicate this effect when considering the features of modular ontologies, it would be a great step forward in optimisation of ontology mapping and creating a new level of expression those systems would work on.

\section{The extension of modularity application in novel approaches}

There are proposals that employ modularity and knowledge representation concepts into artificial neural network. ${ }^{4}$ Moreover, the notion of interweaving neural networks and knowledge representation systems was proposed in regard to ontology mapping. ${ }^{5} \mathrm{We}$ have

4 I. Kollia and others, Interweaving knowledge representation and adaptive neural networks, "Workshop on Inductive Reasoning and Machine Learning on the Semantic Web" 2009.

5 Y. Peng, Ontology mapping neural network: an approach to learning and inferring correspondences among ontologies, Pittsburgh 2010. 
yet to see a proposal that would employ any form of modularity in a meaningful way into those two venues of scientific discourse. Both of mentioned claims are concerned with hybrid structure, where the architecture of the system is very complex. A question addressing this complexity can arise: would such systems benefit from modular structure? In the response to interweaving knowledge representation system and ANN the answer is yes, but in regard to the second claim, the question remains open. Although when we consider the possibility of utilizing emergent functions, then both of those claims would certainly benefit from it, because of the economy of function implementation that would be inherent and solely caused by structurality of the system itself. There is another issue at stake here: to what extent we can depend on the stability of emergent functions in artificial systems? The all-encompassing ability to autoregulate features of biological systems is prevalent in a vast majority of them, whereas in artificial structures such effortless implementation to this day is unachieveable. Despite its shortcomings, the notion of using biological structurality as inspiration for innovative solutions in knowledge engineering, may still provide an insight into how can we utilize some facets of the emergent functions and the scope of advantages of modularity implementation in general. Cognitive science, as interdisciplinary field that is strongly connected with notions of modularity, application of ANN and knowledge representation systems would mostly benefit from such research and emphasis on aforementioned properties.

To some extent the inherent symmetry between proposed theories of modularity of neural networks in the brain, the architecture of the knowledge representation systems and the content of ontologies may seem like a simplistic idea, yet its execution is far more problematic than the way it appears at first glance. In a way, it can respond well to the claim of massive modularity, but on structural level of implementation. ${ }^{6}$ But at this point, one cannot be entirely adamant of the scope of modularity that may be most beneficial in regard to innovative solutions in cognitive science. In one way, aside

6 P. Carruthers, The architecture of mind, Oxford 2006. 
from problems concerning modularity implementation in knowledge engineering, similar case can be witnessed in the field of neural simulation research and Biocomputing. ${ }^{7}$ The need to creating architecutre capable of greater functionality as well as better performance in the process of simulation neural connections, hydrodynamics, cell signalling pathways and more. The problem here is that older, more developed and robust programs (NEURON, GENESIS for instance) that began as compartmentalized toolbox equipped with certain functions that eventually became more akin to monolithic systems than anything resembling at very least a quasi-modular structure. The answer for that was creation of federated approach to their design, and as it can be seen the compartments of the systems are becoming more and more similar to modules, just as new tools are being incorporated into their architecture. ${ }^{8}$ Because of that, alternatives emerge and become an aid in contemporary approaches to brain mapping and simulation of neural structures as well as (in regard to its inherent modular architecture) biocomputation. Such systems are designed from ground up with modularity in mind. One of such projects, called Geppetto is one of constituents of OpenWorm initiative. ${ }^{9}$ The undeniable pluses of this platform are various, but what is most striking is its accessibility (its a web-based platform), as well as ability to monitor and influence the simulations ad hoc, with results and feedback seen immediately after the changes to the simulation are made, whether it is a change in the conductance density or gating variables. The platform also enables the user to pause the simulation at any given point to check the data and make changes in the console by using Standard ML language as its primary tool of interfacing with

7 H. Cornelis, A.D. Coop, J.M. Bower, A federated design for a neurobiological simulation engine: the CBI Federated Software Architecture, "PloS One" (2012) 7 (1), doi: http://dx.doi.org/10.1371/journal.pone.0028956.

8 An exampe of that would be the possibility to employ python as a primary language for user-defined scripts as well as a medium for better software component integration. H. Cornelis, and others, Python as federation tool for GENESIS 3.0., "PloS One” (2012) 7 (1), doi: vhttp://dx.doi.org/10.1371/journal.pone.0029018.

9 http://www.geppetto.org/ (21.07.2015). 
simulation (aside from contextual menu that is basic, straightforward and intuitive).

All the aforementioned features were implemented because at its core, Geppetto is a modular platform. That allowed the creators to prepare simulation software that embraces one of the main strong points of modular structure - functional versatility. However, the program is in its early stage of development and it is hard to predict what its final version will perform. One thing is certain, that the case of Geppetto shows a divergence from the usual path that such research projects take - most of simulation software employs monolothic structure. Here we can witness a change in the approach that is rarely seen, because of the complexity that might become overwhelming to the developers. Despite that, their effort shows how modular architecture implementation can be advantageous.

\section{Philosophical approach as a step towards interdisciplinarity}

With these arguments in mind, the biggest problem lies within the confines of biological research. How does one find suitable examples of modular structurality that is relevant to implementation in interdisciplinary field? I believe that philosophical analysis may provide at least a suitable guideline for creating a working positive heuristic that will enhance the process of selection. One can say that philosophy contributes vastly to the interdisciplinary character of contemporary scientific research. ${ }^{10}$ Overcoming the metaphysical perspectivism, that encompasses not only a single venue of reason, but above all reason itself, can provide a better understanding to the problem of finding suitable forms of structurality that could be used in functional enhancement of many tools used in cognitive science, as well as knowledge engineering and ontology design.

10 Anniversary issue of „Science“ (125th) lists philosophical problems still at the top of their list of important questions that require a proper scientific explanation. 
Projects entertaining the idea of hybridity in the design of their systems very often mention modularity both on the level of functionality and architecture. In one way or the other, this notion is at times the most viable way of preserving functionality versatility and stability, at the cost of complexity. But, in order to step away from the complex theories of modularity there is one case where modules are not to be designed, but calculated. This theory allows to keep the system in a dynamic modus operandi without the need to remotely embed it into its mechanisms. In connectomics, modularity can be traced by using standard graph and network measurements. Recent studies show that there is correlation between active (cytoarchitecturally defined) brain areas and regions that exhibit larger connectivity as measured by degree centrality. ${ }^{11}$ This finding allows one to see the viability of applying network metrics into biological structures, which in turn can become waypoints toward better understanding of dynamically shaped modularity in biological system. Furthermore, there is no need to limit this kind of analysis to only neural networks, as these tools stem from mathematical approach used in social network theories.

When extrapolated, biological networks centrality measurements shift the area of research from neuroscience further into biocomputation. This opens possibilities for using different modular structures to be analysed and measured in the same manner as neural structures in connectomics. While the possibilities seem vast, this is where the core problem of such research can be outlined - how can we identify viable and interesting modular structures that can enhance the expressivity and functionality of modular ontologies, or in broader spectrum, modular artificial knowledge representation systems? There is a chance that pinpointing structures that support emergent functions and other emergent features should be considered as the most probable sources of insight into the phenomena of

11 E.J. Behrens, O. Sporns, Human connectomics, "Current Opinion in Neurobiology" 22 (2011) 1, pp. 144-153.

P. Hagmann and others, Mapping the structural core of cerebral cortex, "PloS Biology" 6 (2008), doi: http://dx.doi.org/10.1371/journal.pbio.0060159. 
emergence as well as new applications for modularity in cognitive science, biocomputation and for the most part knowledge engineering.

If one is willing to entertain the idea that mind is an emergent property of the brain, then the idea of biologically inspired modular networks with emergent properties becomes even more interesting. If mind, being a very complex phenomena, could be traced to it's emergent origin, then how many other, less intricate features, mechanisms and functions could be embedded into neural structure of the brain? Whether considering reductionist or holistic approach to mind, the sole potential would be a promising beginning for research concerning other, less intricate functions.

\section{Emergence as a quality of complex relational structures}

Recent study about the origin of consciousness shows that at this point in time state-of-the-art technology is unable to localize a single structure, or region that would be responsible for it's existence. ${ }^{12}$ Rather than that, conscious perception stems from the global increase in connectivity across the whole brain. It seems that in order to keep the modular approach in this case, one would need to resort to connectomics. Dynamic modularity, embedded in network structure is an idea which in the light of recent findings, can show that fixed modular structure, with highly defined modules according to broadly accepted guidelines, may be insufficient foundation for vastly diversified functionality found in neural networks. Furthermore, this diversification of functions is not limited only to brain structures, but can also be applied to analysis of metabolic tracts of protein kinases and their artificial counterparts found in biocomputational approaches used in modelling of these phenomena. Treating modularity as a concept that can be calculated rather than defined

12 D. Godwin, R. L. Barry, R. Marois, Breakdown of the brain's functional network modularity with awareness. "Proceedings of the National Academy of Sciences of USA" 112 (2015) 12, pp. 3799-3804, doi: 10.1073/pnas.1414466112. 
and implemented allows one to apply its core structural characteristics in various research fields. In actuality, most structures possessing a network or graph structure can be subjected to such analysis and refurbishing in order to alter its functionality. Still, there is a need for research in this field, as we still need a better understanding of emergent properties in general.

Although application of emergent properties and functions it is a substantial problem, there are already ways to apply emergent properties in area of informatics. There are methods of optimization that stem from very basic phenomena related to structurality and emergent function. Metaheuristic, called particle swarm optimization (PSO) is an algorithm that is based on swarm intelligence that is a property most often found in biological systems and can be extrapolated into theories concerning foundation of social dynamics. ${ }^{13}$ Using simulations of bird flock movement authors of particle swarm optimization created an algorithm that would create a swarm of agents that moved acoording to few simple principles. To quote the authors, a simple application, taken from behaviour of biological life-forms allowed to create "synchrony of movement." Later, that algorithm needed other variables to become useful tool for optimization, like craziness. It allowed the function to achieve greater stability in order to stave off inevitable convergence of particles. There are many versions and updates on the subject of PSO, but one thing is shared among all iterations of this method: simple functioning agents create a new feature that they respectively lack - intelligence that would be impossible to achieve if we would treat them separately.

In conclusion, it is worth noting that contemporary theories of modularity are becoming divided between quasi-traditional approach that can be traced to Fodor's idea of modularity and connectomics-driven one, that is based on the principles of centrality, networking, and graph structure. Still, this topic needs further examination as empirical work in this field needs more time to accumulate more knowledge concerning structural connectivity and functional plas-

13 J. Kennedy, R. Eberhart, Particle swarm optimization, Piscataway, NJ. 1995. 
ticity on various levels of scientific cosideration. Viability of newer theories associated with connectomics is unqestionable, but the perspective of finding more about structures with emergent functions and later implementing them to knowledge representation systems could vastly improve functionality and expressivity of those systems. At this point in time there are clues that point out that phenomena associated with systems biology, biocomputation can additinally help in bridging the gap between the mind and brain, by providing answers to emergence of functions and properties that govern both the mind and body.

\section{Summary}

\section{Modular structurality and emergent functionality within knowledge representation systems}

There are various approaches to ontology metamodelling, and the notion of biologically inspired modular knowledge representation systems can provide insight in the workings of such phenomena as emergent properties of network structures. What is more relevant from knowledge engineering standpoint, such approach could provide innovation and enhancement of the level of expression as well as overall functionality of modular ontologies. To do so, one needs to find biological structures that would be the basis for modularity on different levels of hierarchy within the artificial system. Network analysis tools as well as systems biology and biocomputing provide a framework for research in this field.

Keywords metamodelling, ontology, proteomics, connectomics, centrality, philosophy of information

\section{Bibliography}

Azam F., Biologically inspired modular neural networks, Blacksburg, VA 2000.

Behrens E. J., Sporns O., Human connectomics, "Current Opinion in Neurobiology" 22 (2011) 1, pp. 144-153.

Carruthers P., The architecture of mind, Oxford 2006

Cheng F. and others, Quantitative network mapping of the human kinome interactome reveals new clues for rational kinase inhibitor discovery and 
individualized cancer therapy, "Oncotarget" 15 (2014), p. 3697-3710, doi: 10.18632/oncotarget.1984.

Cornelis H., Coop A. D., Bower J. M., A federated design for a neurobiological simulation engine: the CBI Federated Software Architecture, "PloS One" (2012) 7 (1), doi: http://dx.doi.org/10.1371/journal.pone.0028956

Cornelis H. and others, Python as federation tool for GENESIS 3.0., "PloS One" (2012) 7 (1), doi: vhttp://dx.doi.org/10.1371/journal.pone.0029018.

Godwin D., Barry R. L., Marois R., Breakdown of the brain's functional network modularity with awareness. "Proceedings of the National Academy of Sciences of USA" 112 (2015) 12, pp. 3799-3804, doi: 10.1073/ pnas. 1414466112 .

Hagmann P., and others, Mapping the structural core of cerebral cortex, "PloS Biology" 6 (2008), doi: http://dx.doi.org/10.1371/journal.pbio.0060159.

Kennedy J., Eberhart R., Particle swarm optimization, Piscataway, NJ. 1995.

Kollia I. and others, Interweaving knowledge representation and adaptive neural networks, "Workshop on Inductive Reasoning and Machine Learning on the Semantic Web" (2009) 12, pp. 1-4.

Peng Y., Ontology mapping neural network: an approach to learning and inferring correspondences among ontologies, Pittsburgh 2010.

Roche C., Network analysis of Semantic Web Ontologies, Stanford CS224W: Social and Information Network Analysis 2011.

Zuo X. N., R and others, Network centrality in the human functional connectome, "Cerebral Cortex" 22 (2012) pp. 1862-1875, doi: 10.1093/cercor/ bhr269. 